\title{
SPECTROSCOPY OF THE LOW-LYING STATES NEAR THE HIGH SPIN ISOMER IN ${ }^{108} \mathrm{Ag}^{*}$
}

\author{
J. Sethi ${ }^{\mathrm{a}}$, R. Palit ${ }^{\mathrm{a}}$, J.J. Carroll ${ }^{\mathrm{b}}$, S. Karamian ${ }^{\mathrm{c}}$, S. Saha ${ }^{\mathrm{a}}$

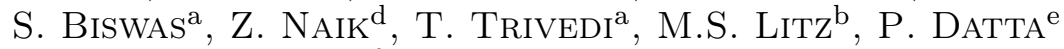 \\ S. Chattopadhyay ${ }^{\mathrm{f}}$, R. Donthi ${ }^{\mathrm{a}}$, U. GarG ${ }^{\mathrm{g}}$, S. JAdhaV ${ }^{\mathrm{a}}$

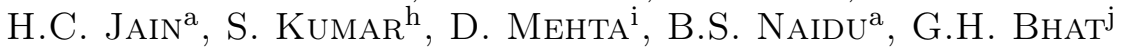 \\ J.A. Sheikh ${ }^{\mathrm{j}}$, S. Sihotra ${ }^{\mathrm{i}}$, P.M. WALKER ${ }^{\mathrm{k}}$ \\ aTata Institute of Fundamental Research, Mumbai 400005, India \\ ${ }^{b}$ U.S. Army Research Laboratory, Adelphi, MD 20783, USA \\ c Joint Institute for Nuclear Research, Dubna 141980, Russia \\ ${ }^{\mathrm{d}}$ Sambalpur University, Sambalpur 768019, India \\ eAnanda Mohan College, Kolkata 700009, India \\ ${ }^{\mathrm{f}}$ Saha Institute of Nuclear Physics, Kolkata 700064, India \\ guniversity of Notre Dame, Notre Dame, IN 46556, USA \\ ${ }^{\mathrm{h}}$ University of Delhi, Delhi 110007, India \\ ${ }^{i}$ Panjab University, Chandigarh 160014, India \\ ${ }^{j}$ Department of Physics, University of Kashmir, Srinagar 190006, India \\ ${ }^{k}$ Department of Physics, University of Surrey, Guildford, Surrey GU2 7XH, UK
}

(Received January 21, 2015)

A comprehensive study of the low-lying states of ${ }^{108} \mathrm{Ag}$, near the isomeric state at $E_{\mathrm{i}}=110 \mathrm{keV}$ with $J^{\pi}=6^{+}$and $T_{1 / 2}=438 \mathrm{y}$, has been presented. Spectroscopy of these states has been carried out using the reaction ${ }^{100} \mathrm{Mo}\left({ }^{11} \mathrm{~B}, 3 n \gamma\right){ }^{108} \mathrm{Ag}$ at $39 \mathrm{MeV}$ beam energy using INGA. The multipolarities and electromagnetic nature of the transitions have been assigned based on the angular correlation and polarization measurements. The experimentally identified states have been compared to the results of the Projected Hartree-Fock calculations to understand the configurations involved in these states.

DOI:10.5506/APhysPolB.46.703

PACS numbers: 21.60Jz, 27.60. $+\mathrm{j}, 23.20 .+\mathrm{g}, 23.20 .-\mathrm{g}$

\section{Introduction}

Study of the long lived excited states of nuclei, known as isomers or metastable states, has always been a dynamic field of research in nuclear

* Presented at the Zakopane Conference on Nuclear Physics "Extremes of the Nuclear Landscape", Zakopane, Poland, August 31-September 7, 2014. 
physics. The hindered decay of these isomeric states may arise due to different reasons, for example, the delayed transitions may occur due to shape changes, large spin difference or large difference in $K$-values [1]. The nuclear chart has a great assortment of isomers with lifetimes ranging from a few nano-seconds to hundreds of years but only a few of these isotopes have half-lives greater than a day [2]. The metastable states are storehouses of energy in the range of $\sim 100 \mathrm{keV}$ to a few $\mathrm{MeV}$. The very long lived isomers having lifetimes longer than years are particularly interesting because of their possible applications for the development of energy storage devices. Excitation of the isomeric state and the subsequent de-excitation through a depleting pathway motivates the research initiatives to harness the energy stored in the isomer through controlled emission.

The aim of the present investigation was to search for more depletion paths in ${ }^{108} \mathrm{Ag}$, near the $J^{\pi}=6^{+}, E_{\mathrm{i}}=110 \mathrm{keV}$, with low relative excitation energies compared to the isomeric state. The spectroscopic information of the states near this isomer is very much essential to evaluate the capability of the nuclei to make transitions to and from the metastable state. The experimental study of the structure of low-lying states of ${ }^{108} \mathrm{Ag}$ has been presented and compared to Projected Hartree-Fock calculations.

\section{Experimental set-up and analysis details}

The excited states of ${ }^{108} \mathrm{Ag}$ were produced in an experiment using the reaction ${ }^{100} \mathrm{Mo}\left({ }^{11} \mathrm{~B}, 3 n \gamma\right){ }^{108} \mathrm{Ag}$ at $39 \mathrm{MeV}$ beam energy. The $\gamma$-rays from the de-excitation of the nucleus were detected using the Indian National Gamma Array (INGA) comprising of 18 Compton suppressed clover HPGe detectors. Two- and higher-fold coincident data were recorded in the Pixie16 digital data acquisition system [3]. The time stamped data were sorted using the routine Multi pARameter time-stamped based COincidence Search program (MARCOS), developed at TIFR, to generate $E_{\gamma}-E_{\gamma}$ matrices and $E_{\gamma}-E_{\gamma}-E_{\gamma}$ cubes, which were analyzed using the RADWARE package to build the level scheme [4]. The multipolarities of $\gamma$-rays were deduced from the angular distribution and angular correlation analysis using the Directional Correlation of Oriented states of nuclei (DCO) ratio method [5]. The parities of the states were deduced from the polarization asymmetry measurements $[6,7]$. The details of the analysis can be found in Ref. [8].

\section{Results}

A detailed spectroscopy of the states in ${ }^{108} \mathrm{Ag}$ nucleus have been carried out [8]. A total of 65 new $\gamma$-transitions have been added to the level scheme based on two- and higher-fold coincidence analysis, in addition to confirmation of the previously reported level structure by Espinoza-Quiñones et al. [9]. 
The spin-parity assignments for most of the levels have been established from the DCO and polarization asymmetry measurements. For the lowlying states, which are of special interest here, 10 new $\gamma$-rays and a few new levels have been identified. We identified three intermediate states with excitation energies less than $420 \mathrm{keV}$, viz., with 255, 358 and $413 \mathrm{keV} \gamma$-rays, which have decay branches to isomeric state and also to the ground state. The states near to the isomer are shown in the partial level scheme given in Fig. 1. The spectrum depicted in Fig. 2 generated with double gate on 55 and $215 \mathrm{keV}$ transitions shows the $\gamma$-rays near and above the isomer. The energies we need to excite the isomer (shown with upward arrows in Fig. 1) to one of these intermediate states (IS) whose subsequent decay would release a total energy $E_{\text {tot }}=E_{m \rightarrow \mathrm{IS}}+E_{m}+Q_{\beta}$, where $E_{m \rightarrow \mathrm{IS}}$ is the excitation of the isomeric state to one of the IS, $E_{m}$ is the energy trapped inside the isomeric state and $Q_{\beta}$ is the energy of the $\beta$-decay of the ground state. For the ${ }^{108} \mathrm{Ag}$ nucleus, $E_{m}=110 \mathrm{keV}$ and $Q_{\beta}=1.65 \mathrm{MeV}$ [10], so the total energy released in depletion of the isomer would be $1.75 \mathrm{MeV}$.

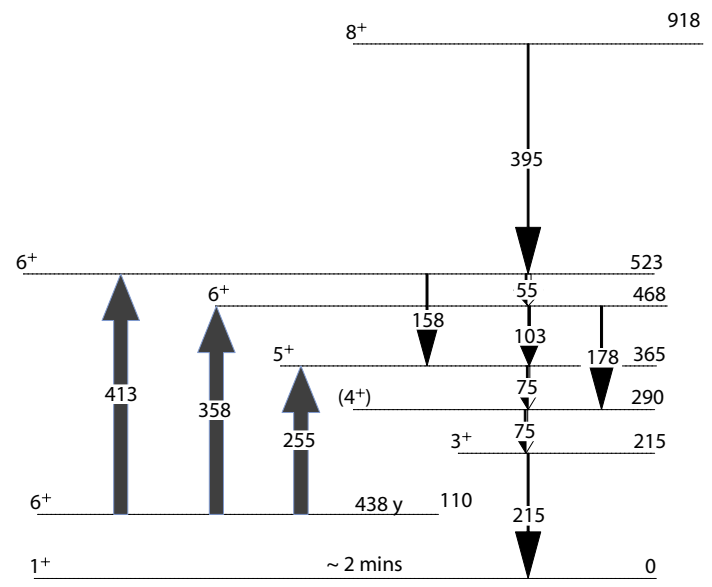

Fig. 1. Partial level scheme of ${ }^{108} \mathrm{Ag}$ showing the levels near the $J^{\pi}=6^{+}$isomer and the identified depletion pathways.

To estimate the cross section for the excitation of the isomer to an IS, followed by the decay of IS to the ground state, requires a thorough investigation of the spectroscopy of these states. The level energies, $\gamma$-ray intensities, total branching ratios, $\gamma$-branching ratios, spins and parities of these levels have been measured. Using the experimental spectroscopic information, integral cross sections have been estimated for the isomer depletion via these intermediate states [11]. We confirmed the $J^{\pi}$ assignments for most of the levels near the isomer. The spin parity determination for ${ }^{108} \mathrm{Ag}$, being an odd-odd nucleus, is complicated because of a number of closely 


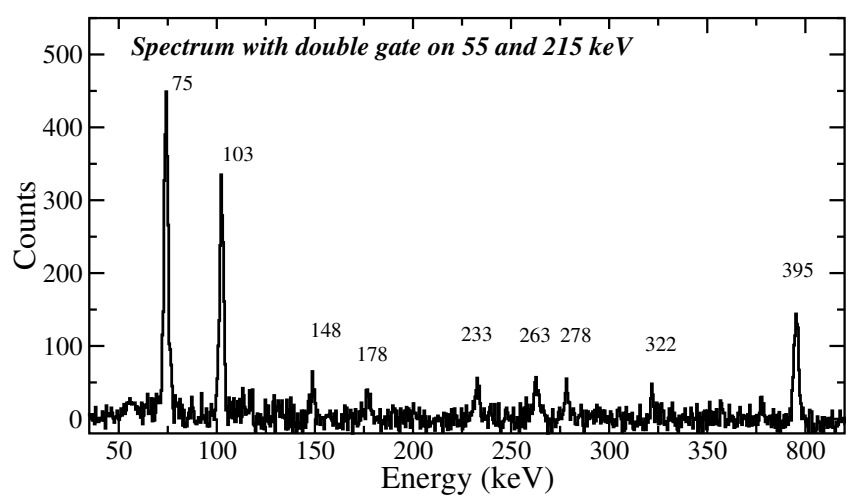

Fig. 2. Spectrum with 55 and $215 \mathrm{keV}$ double gate, showing various transitions $75 \mathrm{keV}$ doublet, 103, 178 and $395 \mathrm{keV}$ near the isomer.

lying states with different possible $J^{\pi}$. We carried out a deformed projected Hartree-Fock (PHF) calculations for these states to understand different configurations giving rise to these levels. From the prolate Hartree-Fock mean field solution with a deformation $\beta=0.15$, these low-lying states of ${ }^{108} \mathrm{Ag}$ were found to have contributions from $\pi g_{9 / 2}$ and $\nu g_{7 / 2}$ orbitals. Seven rotational bands, based on 2-quasi-particle coupling, for different $K$-values were calculated below $1.5 \mathrm{MeV}$ excitation energy. The comparison of the PHF calculated values to the experimental results have shown that different levels near the isomer arise from different configurations and it also reproduced the experimentally observed spin-parity values for these states.

\section{Conclusion}

The low-lying states of ${ }^{108} \mathrm{Ag}$ were studied in detail. Significant spectroscopic information about these states have been deduced. The intensities of the $\gamma$-rays, branching ratios, spins and parities of the levels have been assigned. The present measurement will be helpful in improving the estimation of cross section for the depletion of the isomer. The Projected Hartree-Fock calculations have been performed and compared to the experimental results to understand the underlying configurations of these states.

The authors would like to thank the members of INGA PICC and INGA Collaboration and the accelerator staff at TIFR-BARC Pelletron-LINAC Facility. This work was partially supported by the Department of Science and Technology, Government of India (Grant No. IR/S2/PF-03/2003-II). 


\section{REFERENCES}

[1] P.M. Walker, G.D. Dracoulis, Nature 399, 35 (1999).

[2] J.J. Carroll, AIP Conf. Proc. 1109, 44 (2009).

[3] R. Palit et al., Nucl. Instrum. Methods Phys. Res. A680, 90 (2012).

[4] D.C. Radford, Nucl. Instrum. Methods Phys. Res. A361, 297 (1995).

[5] A. Krämer-Flecken et al., Nucl. Instrum. Methods Phys. Res. A275, 333 (1989).

[6] K. Starosta et al., Nucl. Instrum. Methods Phys. Res. A423, 16 (1999).

[7] R. Palit et al., Pramana 54, 347 (2000).

[8] J. Sethi et al., Phys. Lett. B725, 85 (2013).

[9] F.R. Espinoza-Quiñones et al., Phys. Rev. C52, 104 (1995).

[10] http://www.nndc.bnl.gov/nudat2/ decaysearchdirect. jsp?nuc=108AG\&unc $=$ nds

[11] J. Sethi et al., submitted to Phys. Rev. C (2014). 